\title{
Wie wirksam ist das Fortbildungsprogramm «Patientenseminar Angst»? Ein erster Erfahrungsbericht
}

\author{
How Effective Is the Patientseminar 'Anxiety'?
}

\author{
A. Perkonigg, H. U. Wittchen \\ Max-Planck-Institut für Psychiatrie, Klinisches Institut, Klinische Psychologie, München
}

\begin{abstract}
Zusammenfassung und Schlüsselwörter
Der folgende Beitrag schildert erste Ergebnisse einer Evaluationsstudie des Fortbildungsprogramms «Patientenseminar Angst» für Ärzte. 109 Teilnehmer der Veranstaltung I und 104 Teilnehmer der Veranstaltung II wurden vor und im AnschluB an die Veranstaltungen sowie 3 Monate später bezüglich ihrer Beurteilung des Fortbildungsprogramms sowie zu ihren Einstellungen, Erfahrungen und Kenntnissen zu Angstpatienten befragt und mit einer Kontrollgruppe verglichen. Es zeigte sich, daß Angstpatienten in der Allgemeinarztpraxis viel Zeitaufwand beanspruchen und sowohl die diagnostischen als auch die therapeutischen Kenntnisse nicht zufriedenstellend sind. Das Fortbildungsprogramm wurde von den Teilnehmern sehr gut beurteilt. Durch die Teilnahme wurde in Teilgruppen eine Verbesserung vor allem der Kenntnisse im Bereich «Angststörungen erkennen/Diagnostik» erreicht. Das Patientenseminar wurde vom Großteil der Teilnehmer sowohl in Gruppen als auch bei einzelnen Patienten in der Praxis durchgeführt. Es wird deutlich, daß trotz des hohen Aufwandes eine große Akzeptanz für den Einsatz solcher Patientenseminare vorhanden ist und $\mathrm{da} B$ insgesamt die bisher untersuchten Variablen für eine überraschend hohe Effektivität der Fortbildungsmaßnahmen sprechen.
\end{abstract}

Angststörungen, Diagnostik und Therapie Medizinische Fortbildung, Evaluation Allgemeinmedizinische Versorgung

\section{Summary and Key Words}

This article describes preliminary results of an evaluation study of the patientseminar 'anxiety', an educational programme for physicians. Before and after the two seminar meetings, 109 participants of part I and 104 participants of part II filled in a questionnaire about their opinion on the programme as well as their attitudes, experience, and knowledge about anxiety patients. They were compared with a control group, which did not take part in the programme. It is found that anxiety patients call for a lot of attention at general practitioners' and that the physicians' diagnostic and therapeutic knowledge is not sufficient. The educational programme was highly rated by participants. The patientseminar was conducted, for the greater part of participants, by the physicians both in groups and for single patients. It became evident that in spite of high expenses there is a wide acceptance of such patientseminars and that the variables so far examined up to now indicate surprisingly high efficiency of the educational programme.

Anxiety disorders, diagnosis and therapy - Medical education programme, evaluation. Primary care

\section{Einleitung}

Angststörungen sind weitverbreitete und lang andauernde, oft chronische Störungen. Viele epidemiologische Untersuchungen haben in den vergangenen Jahren bestätigt, daß Angststörungen (Panikstörung, Generalisierte Angst, Agoraphobie, Soziale Phobien, Isolierte Phobie) die häufigsten Formen psychischer Störungen in der Allgemeinbevölkerung sind und auch in der allgemeinärztlichen Versorgung weit verbreitet sind. Etwa $25 \%$ aller Allgemeinarzt-Patienten klagen über Angstsymptome [Sartorius et al., 1990; Wittchen et al., 1992]. Trotz ihrer Häufigkeit werden Angststörungen in der Routineversorgung oft nicht erkannt, fehldiagnostiziert und/oder fehlbehandelt. Eine noch unveröffentlichte Studie der Weltgesundheitsbehörde [Sartorius et al., im Druck] schätzt für die Bundesrepublik, daß ca. $50 \%$ aller Angststörungen ibberhaupt nicht erkannt oder als Depression

\footnotetext{
${ }^{1}$ mit Förderung der Firmen Upjohn, Heppenheim, und Duphar, Hannover.
}

oder somatische Störungen fehldiagnostiziert werden. Weitere $25 \%$ werden nach Expertenurteil fehlbehandelt. Auf diese Problematik wurde in der Vergangenheit immer wieder hingewiesen und die Notwendigkeit zur Verbesserung der Versorgung dieser Patientengruppe betont [Sartorius et al., 1990; Sartorius et al., in press]. Die Wichtigkeit einer Verbesserung dieser Situation wird besonders evident, wenn berücksichtigt wird, daß viele Patienten mit Angstsymptomen von ihren Schwierigkeiten erstmals in der Sprechstunde ihres Allgemeinarztes berichten und psychologischen, psychotherapeutischen und psychiatrischen Interventionen oft reserviert gegenüberstehen. In der Folge werden diese Patienten oft über viele Jahre hinweg von einem Allgemeinarzt betreut, ohne adäquat diagnostiziert oder behandelt zu werden. Unabhängig hiervon macht es der zum Teil chronische Verlauf von Angststörungen in nicht wenigen Fällen erforderlich, daß der Allgemeinarzt - u.a. aufgrund mangelhafter Kenntnisse zu bestehenden Therapiemöglichkeiten - diese Patienten über Jahre hinweg stützen und beraten muß, ohne differenzierte Therapieangebote $z u$ machen. Allerdings wird diese Art von Betreuung der Angstpatienten offensichtlich von 
vielen Allgemeinmedizinern als Belastung empfunden. Gleichzeitig klagen viele Ärzte darüber, daß sie nicht wissen, wie sie mit Angstphänomenen diagnostisch umgehen sollen und welche Behandlungsmöglichkeiten ihnen auch auf dem Uberweisungswege zur Verfügung stehen. Empirisch gestützte Daten zu dieser Frage liegen allerdings derzeit noch nicht vor.

Vor allem Allgemeinärzte stehen damit als häufigste erste Anlaufstelle vor der Aufgabe, neu auftretende Angsterkrankungen 1) frühzeitig und verläßlich zu erkennen, 2) die Patienten zu informieren und aufzuklären und 3) eine Entscheidung über eine Intervention zu treffen; z.B. in Form einer Uberweisung zum Spezialisten oder dem Versuch, selbst pharmakologisch oder psychotherapeutisch $\mathrm{zu}$ intervenieren.

Das im folgenden beschriebene Fortbildungsprogramm "Angst erkennen, diagnostizieren und behandeln" wurde entwickelt, um diese mangelhafte Versorgungssituation $z u$ verbessern. Primäre Zielsetzung des Programms ist, über ein verhaltensmedizinisch orientiertes Fortbildungskonzept die Kompetenz von Allgemeinmedizinern zu verbessern, Angststörungen $2 u$ erkennen und zu diagnostizieren sowie allgemein ihren Wissensstand über neue therapeutische Methoden zu verbessem. Damit wird die Erwartung verbunden, daß Allgemeinärzte Angststörungen:

1) frühzeitiger erkennen,

2) Patienten besser und adäquater beraten,

3) ggf. schneller an den Spezialisten zur Behandlung überweisen,

4) weniger häufig unnötige labortechnische Untersuchungsmaßnahmen durchführen,

5) weniger häufig selbst inadäquate Therapiestrategien anwenden (z. B. zu niedrig dosierte Antidepressiva-Therapie, zu langfristige Benzodiazepin- und Neuroleptikaverschreibungen).

Darüber hinaus wurde bei der Entwicklung darauf geachtet, daß die Materialien praxisnah, konkret und leicht anwendbar sind, sowie auch für Nervenärzte, Psychologen und Psychotherapeuten im Rahmen von Beratung und Therapie geeignet sind.

Um diese Zielsetzungen $z u$ erreichen, wurde von in der Angstbehandlung erfahrenen Psychologen und Ärzten - mit
Unterstützung der Firmengruppen Upjohn und Duphar - ein integriertes zweistufiges Fortbildungskonzept (siehe Tab. 1) konzipiert, das sowohl theoretische (Teil 1) wie auch praktische Übungen (Teil 2) mit konkret ausformulierten Manualen (Patientenseminar Angst) umfaßt. Ergänzend hierzu wurden auf den Fortbildungsinhalt abgestimmte Patientenmaterialien (Ratgeber «Angst») verfaßt, die vom Arzt zur weitergehenden Information einzelner Patienten und ihrer Angehörigen eingesetzt werden können. Diese umfassende zweistufige Fortbildungsstruktur sollte einerseits den Lerneffekt didaktisch absichern, andererseits durch praktische Übungen den späteren Einsatz in der Praxissituation sicherstellen.

Das Fortbildungsprogramm (Tab. 1) wurde in zwei getrennten halbtägigen Veranstaltungen durchgeführt: In der Veranstaltung I wurden zunächst die Grundlagen und Neverungen der modernen differenzierteren Angstdiagnostik und Therapie vermittelt. Dazu wurde den Teilnehmern ein Kompendium; "Diagnostik und Therapie von Angststörungen - Ein Handbuch für den Arzt. [McGlynn \& Metcalf, 1993] vorgestellt und in einem Vortrag veranschaulicht. Danach hatten die Teilnehmer Gelegenheit zur Aussprache sowie zur Einübung der Kenntnisse mit einem computerisierten interaktiven Lernprogramm. Letzteres erlaubte es, sowohl die theoretischen wie auch die praktischen Bausteine durch Fragen, Erläuterungen und Fallbeispiele zu vertiefen und den Wissensstand zu überprüfen.

In der etwa drei Monate später folgenden Veranstaltung II wurde dann das Kernstück des Fortbildungsprogramms, das "Patientenseminar Angst: Wie informiere ich meine Patienten über Angst?» [Wittchen et al., 1993], vorgestellt, diskutiert und zum Teil im Rollenspiel praktisch eingeübt.

Derartige Patientenseminare sind als zeitsparende Informationsvermittlung sowie als compliancestitzende Maßnahmen in verschiedenen Bereichen der Verhaltensmedizin schon seit vielen Jahren weit verbreitet. So z. B. bei Stoffwechselerkrankungen (Diabetes) oder in der Orthopädie (Rückenschule), aber auch in der Verhaltenstherapie, z. B. von Panikstörungen [Margraf \& Schneider, 1989]. Das Konzept der Patientenseminare besteht darin, einzelne oder Gruppen von Patienten mit ăhnlicher Symptomatik zu einer Informationsveranstaltung einzuladen und über das Störungsbild, die
Tab. 1. Struktur des Fortbildungsprogramms «Angst»

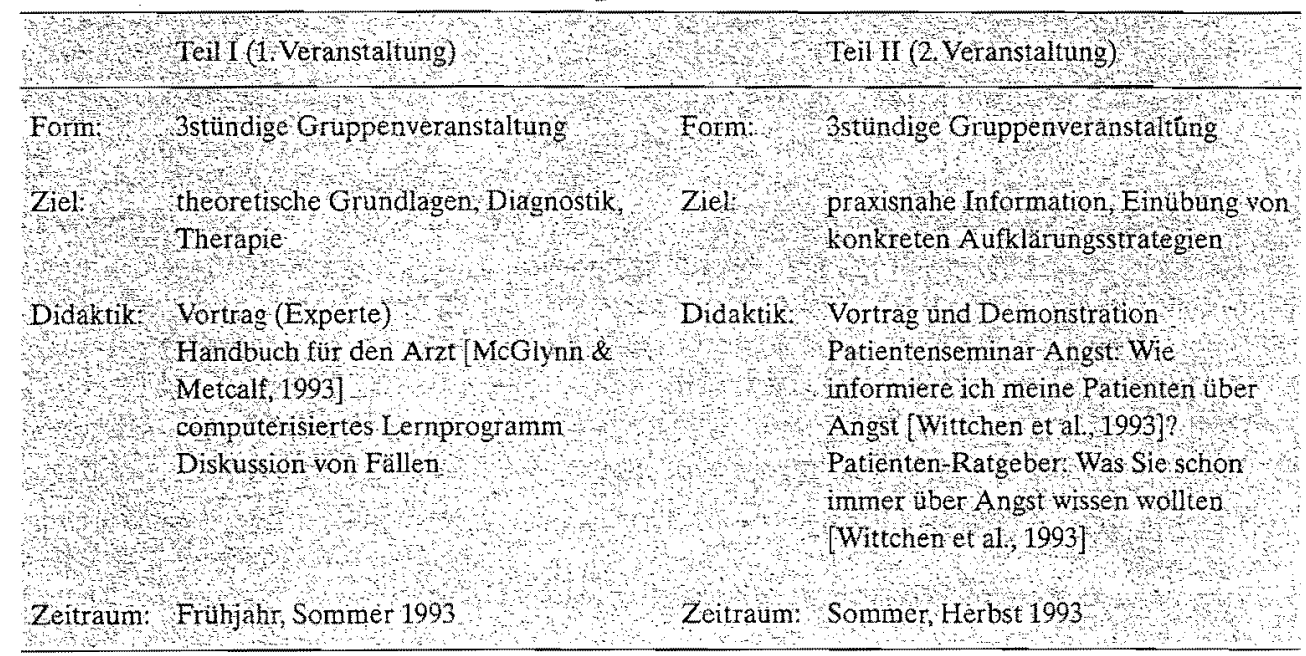


Therapie und die Langzeitprognose aufzuklären und so eine hilfreichere Einstellung der Patienten im Hinblick auf die Therapie und die Spontanprognose zu erreichen. Patientenseminare haben sich als motivierend für den Patienten, entlastend und zeitsparend für den Therapeuten sowie auch als wirksam in Hinblick auf die Prognose erwiesen. Das Patientenseminar Angst enthält in diesem Rahmen neben organisatorischen Hinweisen konkret ausformulierte Textbausteine und Abbildungen (Dias), die in einfacher Form folgende Fragen beantworten helfen: Was ist eigentlich Angst? Warum haben wir Angst? Wie äuBert sich Angst? Wann wird Angst zur Krankheit? Welche Angststörungen gibt es? Wie entstehen Angststörungen? Was geschieht, wenn ich nichts unternehme? Wie können Angststörungen behandelt werden? Daruber hinaus sind im Anhang neben typischen zusätzlichen Patientenfragen mit Antwortmöglichkeiten auch diagnostische Leitfragen zur Diagnostik aufgeführt, die bei der Auswahl der Patienten eine Hilfe sein sollen.

Unmittelbares Ziel der Veranstaltung II war es, einzuüben, mit welchen Worten und Inhalten verschiedene Gruppen von Angstpatienten über ihre Erkrankung und Behandlungsmöglichkeiten adäquat informiert werden können. Indirekt kann diese Veranstaltung aber auch als vertiefende Weiterbildung im Sinne der Veranstaltung I angesehen werden, da die Inhalte konkret in Worte umgesetzt und im Rollenspiel erprobt werden.

Am Ende der Veranstaltung II wurde allen Teilnehmern das komplette Material des Patientenseminars zur Verfügung gestellt, das aus der Seminaranleitung [Wittchen et al., 1993] sowie den Diapositiven für Gruppenveranstaltungen besteht. Ergänzt wird das Programm durch den auf die Inhalte des Patientenseminars abgestimmten Patienten-Ratgeber Angst "Was Sie schon immer über Angst wissen wollten» [Wittchen et al., 1993], der den teilnehmenden Patienten bzw. interessierten Angehörigen das Nachlesen und Nachbereiten der im Seminar vom Arzt/Therapeuten vermittelten Informationen ermöglichen soll.
Es handelt sich also um ein umfassendes Fortbildungskonzept, das versucht, die modernen Prinzipien der medikamentösen, psychotherapeutischen und verhaltenstherapeutischen Therapie bei Angststörungen zu vermitteln.

\section{Fragestellungen}

In folgenden wollen wir erste Ergebnisse einer Evaluationsstudie dieses Fortbildungsprogramms vorstellen. Ziel der Evaluation ist es zu prüfen, inwieweit und speziell welche der Interventionskomponenten zu einer meßbaren Veränderung des Wissens und der Einstellung der teilnehmenden Ärzte gefuhrt haben. Im einzelnen sollen folgende Fragestellungen untersucht werden:

1) Was wissen Allgemeinärzte über Angststörungen, die Ätiologie und die Behandlung, und welche Einstellung haben sie gegenuber Angstpatienten?

2) Welche Veränderungen ergeben sich aufgrund der ersten Fortbildungsveranstaltung, in der die theoretischen Grundlagen mit Hilfe des Handbuchs, eines Vortrags sowie eines computergestützten Lernprogramms vermittelt wurden?

3) Welche Veränderungen ergeben sich und wie beurteilen die teilnehmenden Ärzte die Einführungsveranstaltung zum Patientenseminar Angst, in dem die konkreten Aufklärungsschritte und Modalitäten vermittelt werden, und zu welchen meßbaren Veränderungen in Wissen und Einstellung kommt es insgesamt?

4) Welcher Prozentsatz von Ärzten hat in einem 3-MonatsZeitraum nach Teilnahme am zweiten Seminar das Patientenseminar eingesetzt, und wie beurteilen sie ihre bisherigen Erfahrungen?

In der vorliegenden Auswertung der Pilotphase des noch nicht abgeschlossenen Projekts werden das Gesamtkonzept sowie einzelne Komponenten auf Akzeptanz und Umsetzbarkeit in der Allgemeinarztpraxis überprüft.
Tab. 2. Wie viele Arzte haben an der Fortbildungsveranstaltung in den Untersuchungsregionen teilgenommen?

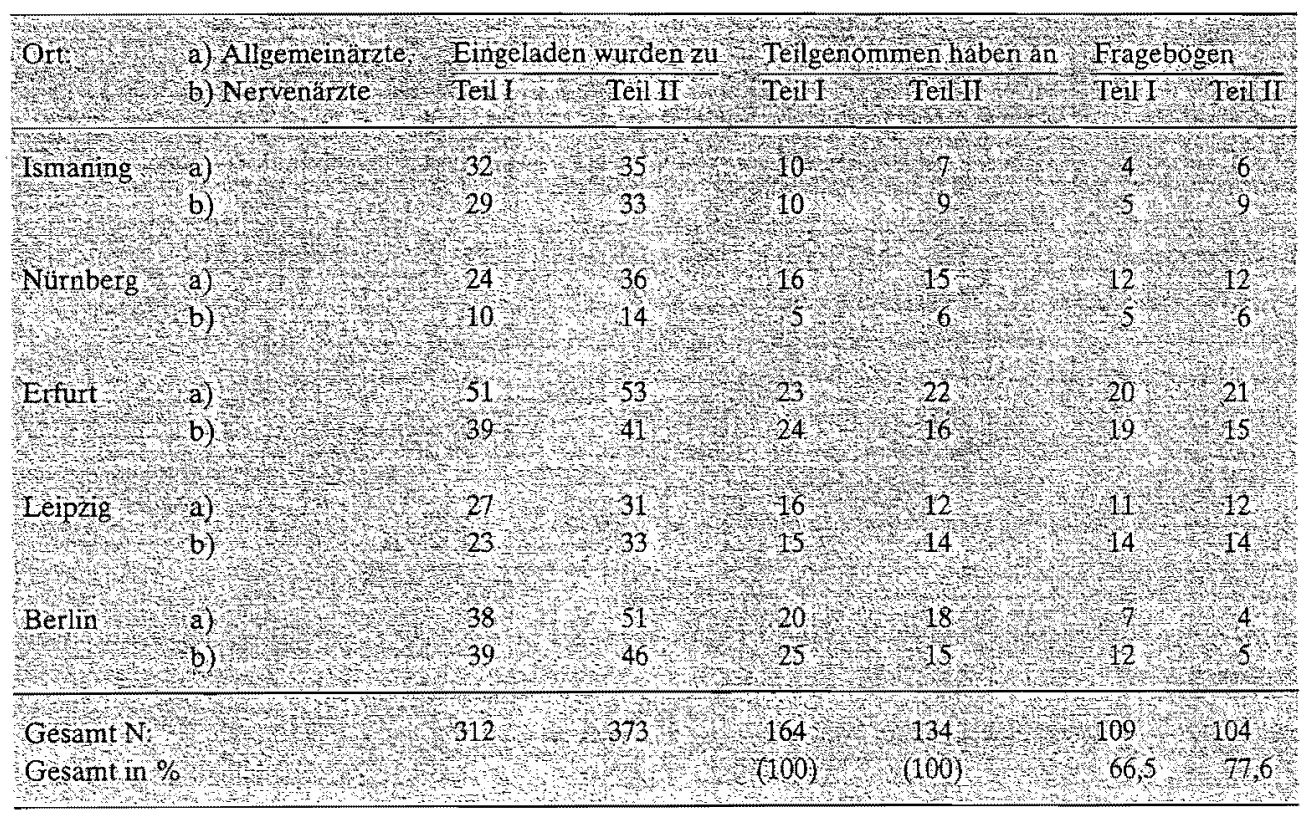




\section{Methodik}

Untersuchungsregionen: Die Evaluationsstudie wurde regionalisiert in folgenden Großräumen durchgefuhrt: Berlin, Leipzig, München, Nürnberg und Erfurt. Dabei wurden über die Pharmareferenten der beteiligten Firmen nach einem Zufallsverfahren erstens Allgemeinärzte und Internisten und zweitens in jeweils getrennten Veranstaltungen Nervenärzte und Neurologen zur Teilnahme eingeladen.

Teilnehmer: Eingeladen wurden insgesamt 312 Arzte zur Veranstaltung I und 373 zur Veranstaltung II. Der Anteil von Nervenärzten und Neurologen betrug $55,1 \%$ (Veranstaltung $\mathrm{D}$ ) bzw. $55,2 \%$ (Veranstaltung $\mathrm{M}$ )

\section{Design der Evaluationsstudie}

Evaluationsmethoden waren Fragebögen und Interview (Interventionsgruppen). Jede der beiden Veranstaltungen wurde mit einem Fragebogen evaluiert. Aus technischen Gründen wurden diese bei der ersten Veranstaltung $\mathrm{z} . \mathrm{T}$. postalisch nach der Durchfïhrung verschickt, während die Fragebögen für die Veranstaltung II direkt am Ende des Seminars ausgehändigt wurden. Teilnehmer, die nur bei der Veranstaltung II teilnahmen, füllten zusätzlich - zur Erhebung von Baseline-Werten - Teile des ersten Fragebogens vor der Veranstaltung aus.

Kontrollgruppe: Die Kontrollgruppe ohne Intervention wurde teilweise brieflich, teilweise telefonisch befragt. Sie wurde aus Machbarkeitserwägungen ausschlieBlich in München untersucht. Dabei wurden per Zufallsverfahren 25 Allgemeinärzte aus dem Branchenbuch ausgewählt. Der Fragebogen für die Kontrollgruppe enthält nur die Teile C, D, E (s. u.).

Fragebögen: Die Fragebögen für beide Veranstaitungen waren gröBtenteils identisch und enthielten neben Fragen z山 Facharztrichtung, Alter, Niederlassungsdauer und Ort folgende Beurteilungsaspekte:

A. Gesambeurteilung: Beurteilung der Güte der Veranstaltung nach Informationsgehalt, Didaktik, Verständlichkeit, Neuigkeitsgrad etc. sowie differenzierte Beurteilung der zusätzlichen Materialien und Unterlagen (Manual, Computerprogramm und Patientenseminar).

$B$. Vorschläge: Was wirde die Teilnehmer weiter interessieren, sowie Verbesserungsworschläge und Nutzen des Seminars.

C. Bisherige Erfahrungen: Wie viele Angststörungen werden in der Praxis diagnostiziert? Welche Diagnosen? Welche medikamentösen und nichtmedikamentösen Verfahren werden bevorzugt eingesetzt? Wie hăufig werden Angstpatienten überwiesen?

D. Einstellung und Wissen: Statements zum Umgang mit Angstpatienten, Statements zur Ätiologie, Diagnostik und Therapie.

E. Fallvignetten: Hier wurden drei Fallgeschichten mit drei unterschiedlichen Angstdiagnosen vorgegeben. $\mathrm{Zu}$ beantworten waren im Multiple-choice-Test, die Hauptdiagnose und indizierte Therapiemaßnahmen (bei letzteren waren Mehrfachangaben möglich).

Follow-up-Untersuchung: Alle Teilnehmer der Veranstaltung II wurden im Durchschnitt 3 Monate nach der Fortbildung telefonisch kontaktiert und gebeten, zu folgenden Fragen Stellung zu nehmen:

Haben Sie seit Teilnahme am zweiten Seminar die Materialien des Patientenseminars Angst verwendet? Wenn ja, bei einzelnen Patienten bzw, bei einer Gruppe? Bei wie vielen Patienten in den letzten Monaten? Wenn das Patientenseminar nicht benutzt wurde, warum? Haben Sie den Eindruck, in der Diagnostik jetzt sicherer als früher zu sein? Erkennen Sie mehr Angststörungen als früher? Fühlen Sie sich in der Therapie von Angststörungen sicherer? Uberweisen Sie häufiger? Wenn ja, an wen?

An dieser Follow-up-Befragung nahmen bislang (Stand: November 1993) 82 von 105 (Responserate: $78,1 \%$ ) Ärzten teil, die den Teil II der Veranstaltung besucht haben.

\section{Ergebnisse}

\section{Teilnahmeraten und Ausschöpfung}

Wie aus Tabelle 2 hervorgeht, folgten 164 von 312 Ärzten der Einladung zur Veranstaltung I und 134 von 373 zur Veranstaltung II. Bezogen auf die Teilnehmer ergibt sich somit eine Ausschöpfungsrate (7. Spalte) von $66,5 \%$ für Veranstaltung I und $77,6 \%$ für Veranstaltung II (Spalte 8). Deutlich wird ferner, daB die Responseraten für Erfurt und Leipzig am höchsten und für Berlin am niedrigsten liegen. Tendenziell weisen auch Nervenärzte eine niedrigere Bereitschaft auf, den Evaluationsbogen auszufüllen.

88 Teilnehmer nahmen sowohl am ersten wie auch zweiten Teil der Fortbildung teil und stehen somit auch für die hier in diesem Artikel noch nicht berücksichtigten Veränderungsmessungen zur Verfügung.

\section{Die Einstellung von Ärxten gegenüber Angstpatienten}

Zu Beginn des Seminars wurden alle Ärzte gefragt, wie sehr sie einer Reihe von Statements zustimmen, die sich auf Angststörungen, den Umgang mit Angststörúngen, ihre Diagnostik, Therapie und Überweisungsmodalitäten beziehen. Auf einer Skala von 1-6 (stimme uberhaupt nicht $\mathrm{zu}-$ stimme voll und ganz zu) ergaben sich dabei folgende Befunde (siehe Abb. 1).

Berücksichtigen wir zumindest leichte Zustimmung als Beurteilungskriterium, so stimmen $91,7 \%$ aller befragten Ärzte der Aussage zu, daß im Vergleich $z u$ anderen Patientengruppen bei Angstpatienten mehr Zeit aufgewendet werden

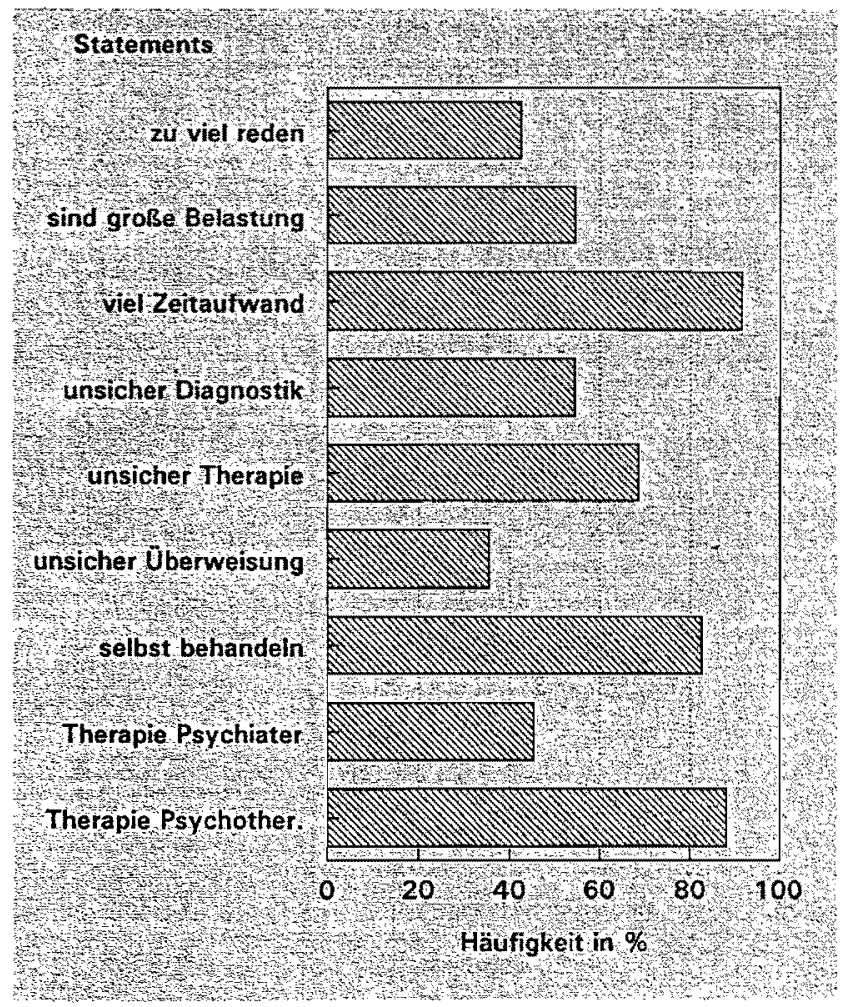

Abb. 1. Einstellungen zu Angstpatienten: Zustimmungsraten (\%) zu obenstehenden Aussagen. 


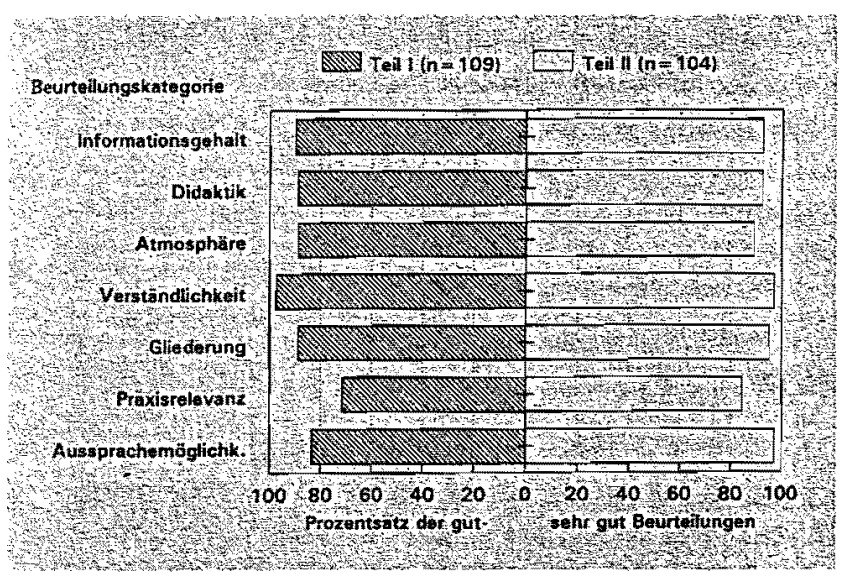

Abb. 2. Wie beurteilen Sie das Patientenseminar Angst Teil I (Theorie) und Teil II (Praxis)?

muß. Zudem geben $68,6 \%$ an, koft unsicher bei der Therapie von Angststörungen zu sein» und $54,4 \%$ «unsicher bei der Diagnostik von Angststörungen zu sein». 54,5\% geben an, $\mathrm{da}$ Angstpatienten keine große Belastung für meine Praxis sind". Mit diesen Befunden korrespondiert, daß $42,4 \%$ der befragten $\ddot{A}$ rzte der Aussage zustimmen, daß Patienten mit Angststörungen zuviel reden wollen. Unabhängig von diesen hohen Zustimmungsraten stimmen aber $82,5 \%$ aller $\mathrm{Be}$ fragten mit dem Statement überein, daB sie Angststörungen in ihrer Praxis selbst behandeln können, gleichzeitig zeigt sich aber auch, daß sie bezüglich der fachspezifischen Therapie häufiger den Psychotherapeuten als zuständig ansehen $(88,2 \%)$ als den Psychiater oder Neurologen $(45,4 \%)$. Ein Drittel aller Befragten ist sich unsicher, wann und ob überhaupt Patienten zu einer fachspezifischen Behandlung überwiesen werden sollen.

\section{Gesamtbeurteilung Zufriedenheit und Einschätzung des Patientenseminars}

Beide Veranstaltungen, sowohl der Teil 1 , in dem mehr die theoretischen Grundlagen, wie auch der Teil 2, bei dem mehr praxisorientiert Aufklärungsstrategien eingeübt wurden, werden von den Teilnehmern als «gut» bzw. «sehr gut» beurteilt (siehe Abb. 2). In allen angeführten Beurteilungskategorien von Informationsgehalt bis Aussprachemöglichkeiten ergeben sich Prozentsätze über $75 \%$ für diese beiden Bewertungen, wobei tendenziell die Beurteilungen der zweiten Veranstaltung etwas höhere Werte aufweisen. Dies gilt insbesondere für den Aspekt «Praxisrelevanz und Aussprachemöglichkeiten», jedoch kann dieser Befund aufgrund möglicher Selbstselektionskriterien für die Teilnahme an der zweiten Veranstaltung auch einen Selektionsartefakt darstellen.

\section{Sicherheit bei der Diagnostik}

Um den Wissensstand der Teilnehmer bezüglich der Differentialdiagnostik einzelner Angststörungen zu überprüfen, gaben wir im Rahmen des Fragebogens drei Fallgeschichten vor, die jeweils auf einer halben Seite die Grundproblematik einer einfachen Phobie, einer Panikstörung und einer posttraumatischen Belastungsstörung zusammenfaßten. Dabei wurde bei der Formulierung der Fallgeschichten darauf geachtet, daß die diagnostischen Kernmerkmale nach DSMIII-R, die Bestandteile der ersten Veranstaltung waren, auch spezifisch und detailliert angesprochen werden. Jede dieser Fallgeschichten sollte von den Teilnehmern diagnostisch eingeordnet werden. Dabei durfte nur eine Diagnose angegeben werden. Die Auswahldiagnosen waren Panikstörung mit und ohne Agoraphobie, Anpassungsstörungen mit ängstlicher Verstimmung, Major Depression, Zwangsstörung, soziale Phobie, posttraumatische Streßstörung, generalisierte Angststörung, einfache Phobie und Psychosen. Darüber hinaus bestand die Möglichkeit, weitere Diagnosen in einer offenen Kategorie einzugeben.

Abbildung 3 gibt zusammenfassend die Prozentraten bezijg. lich der richtigen Diagnosen in der Gesamtgruppe aller Teilnehmer an.

Vor der Durchführung des Patientenseminars ergibt sich eine richtige Trefferrate von $59 \%$ bei der einfachen Phobie, $51,4 \%$ bei der Panikstörung und lediglich $34,3 \%$ bei der posttraumatischen Belastungsstörung. Nach der Durchführung des Patientenseminars mit seinen praktischen Übungen steigt die Trefferrate bezüglich der richtigen Diagnose bei der einfachen Phobie auf 70,2\% an, bei der Panikstörung auf 58,2\% und bei den posttraumatischen Belastungsstörungen auf $46,7 \%$. Bei der Interpretation dieses Befundes muB darauf hingewiesen werden, daß die Trefferrate Befunde» in der Abbildung noch keine Differenzierung nach Psychiater und Nervenärzten versus Allgemeinmediziner ermöglicht. Unter den nicht zutreffenden Diagnosen wurden bezüglich der

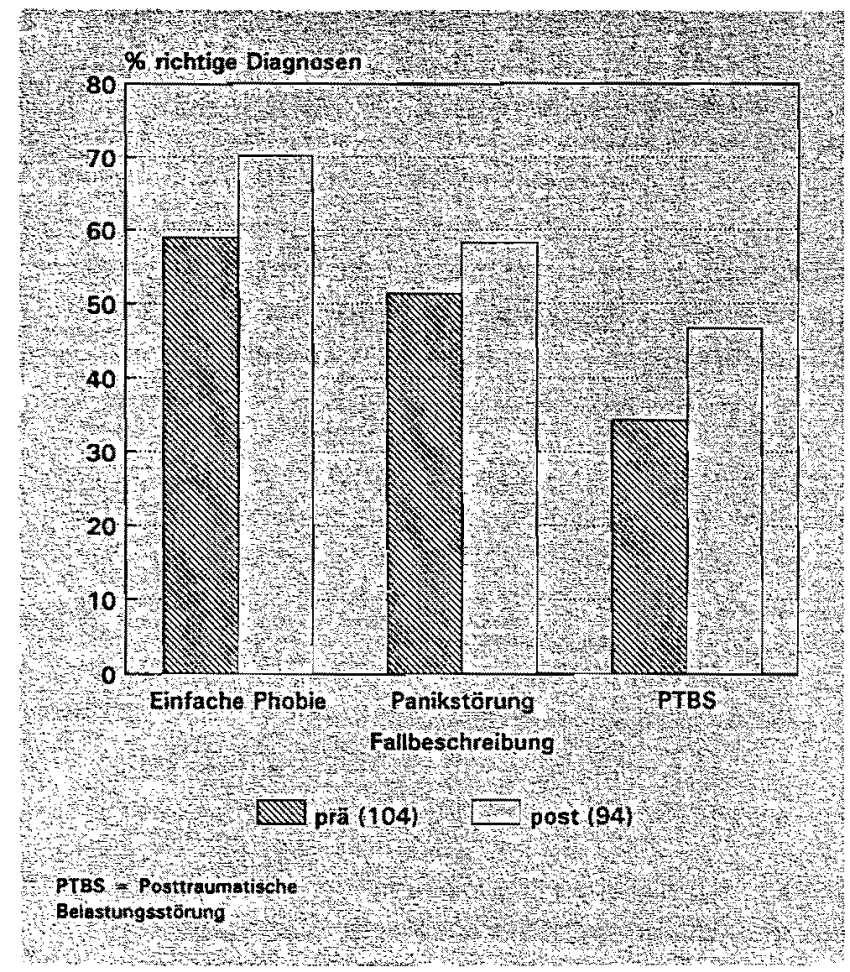

Abb. 3. Können die Teilnehmer Angststörungen richtig diagnostizieren; Trefferrate vor und nach dem Seminar. 
einfachen Phobie am häufigsten posttraumatische Streßstörungen (12\%) sowie Panikstörung (11\%), bezüglich der Panikstörung am häufigsten die generalisierte Angststörung (29\%) und bei der posttraumatischen Streßstörung am. häufigsten eine Major Depression $(30,9 \%)$ diagnostiziert. Besonders auffällig war, daB ein Teilnehmer bei der einfachen Phobie sowie drei Teilnehmer bei der Panikstörung und sieben Teilnehmer bei der posttraumatischen Streß3. störung eine Psychose diagnostizierten.

\section{Effekte des Fortbildungsprogramms und Patientenseminars: Drei Monate später}

Bei der im Durchschnitt drei Monate später durchgeführten telefonischen Nachbefragung ergaben sich folgende Befunde:

\section{Durchführung von Patientenseminaren}

Mehr als zwei Drittel aller Befragten gaben an, die Unterlagen des Patientenseminars zumindest einmal in den vergangenen drei Monaten zur Information und Beratung von Patienten benutzt zu haben. Die Mehrzahl benutzte allerdings die Materialien nicht in einer Gruppenveranstaltung, sondern eher bei einzelnen Patienten. Im Mittel wurden die Unterlagen des Patientenseminars bei 3,8 Patienten eingesetzt. Ein Drittel aller Befragten gab an, Patientenseminare, wie in den Unterlagen vorgegeben, als Gruppenveranstaltung durchgefuhrt zu haben. Dabei betrug die mittlere Gruppengröße 5 Patienten. Teilnehmer, die das Patientenseminar zumindest einmal in einer Gruppenveranstaltung durchführten, gaben auch an, daß mindestens eine weitere Veranstaltung aufgrund des Erfolges durchgeführt wurde. Im Mittel wurde von dieser Gruppe angegeben, daß 9,6 $\mathrm{Pa}$ tienten in den drei Monaten seit Durchführung der Veranstaltung in Gruppenveranstaltungen der Patientenseminare gesehen wurden (siehe Abb. 4).

\section{Erkenner und überweisen}

Auf die Frage, ob die teilnehmenden Ärzte nach der Veranstaltung häufiger Angsterkrankungen in ihrer Praxis erkennen, antworteten $54,9 \%$ aller Betroffenen mit «ja».

$74,4 \%$ gaben darüber hinaus an, häufiger als vor Durchführung des Seminars ihre Angstpatienten zu überweisen. Der gröBte Anstieg war diesbezüglich bei Überweisungen an Nervenärzte festzustellen mit $48,8 \%$. Bemerkenswert sind allerdings auch die angestiegenen Prozentsätze von Überweisungen an Psychologen mit 35,4\% sowie an Psychotherapeuten mit $34,1 \%$ (siehe Abb. 5 ).

\section{Sicherheit in Diagnostik und Therapie}

Auf die abschließende Frage, inwieweit die teilnehmenden Ärzte aufgrund der Fortbildungsmaßnahmen und der überreichten Unterlagen ein höheres AusmaB an Sicherheit in Diagnostik und Therapie erworben haben, ergab sich folgendes Bild. Mehr als drei Viertel aller Teilnehmer haben in Diagnostik und Therapie vom Patientenseminar profitiert. $62,2 \%$ geben an, etwas in der Diagnostik profitiert zu haben,
$12,2 \%$ geben an, mehr davon profitiert zu haben. Bezüglich der Sicherheit in therapeutischen MaBnahmen geben $52,4 \%$ $\mathrm{an}$, etwas sicherer als vor der Veranstaltung $\mathrm{zu}$ sein, $8,5 \%$ sehr viel sicherer. $Z u$ berücksichtigen ist, daß in beiden Beurteilungsaspekten 10,9 bzw. $14,6 \%$ aller Befragten keine Angabe gemacht haben (siehe Abb. 6).

\section{Diskussion}

Die vorliegende Auswertung ist eine erste Vorauswertung der sehr viel differenzierteren Gesamtbefunde. Wir haben uns zu dieser Vorauswertung entschlossen, um einen Beitrag zu der derzeit aktuellen Diskussion über Patientenseminare und Patientenaufklärung zu leisten. Einschränkend zu den Befunden muß angegeben werden, daß sowohl die Kontrollgruppe (ohne jegliche Intervention) wie auch die differenzierteren Auswertungen nach Teilgruppen von Ärzten noch nicht berücksichtigt werden konnten.

Unabhăngig davon zeigen die Ergebnisse deutlich ein außerordentlich hohes AusmaB an Akzeptanz für derartige aufwendige Veranstaltungen, die sich sowohl an der hohen Teilnahmerate wie auch bei der Responserate im Zusammenhang mit den Evaluationsfragebögen und dem Interview zeigt.

Gleichzeitig können wir feststellen, daß die häufig geäußerte Vermutung, daß Angstpatienten eine große Belastung für die allgemeinärztlichen Praxen sind, eindrucksvoll Bestätigung findet. Der hohe Anteil von Ärzten, die darüber klagen, daß Angstpatienten zuviel reden und insbesondere, $\mathrm{d} a \beta$ sie eine große Belastung darstellen bzw. sehr viel Zeitaufwand erfordern, unterstreicht die Notwendigkeit, adäquate Lösungsmodelle zu erarbeiten. Die Handlungsunsicherheit, die in den eingangs zitierten Untersuchungen, u.a. der WHO [1993], deutlich wurden, spiegelt sich auch in der Selbstbeurteilung der befragten Ärzte wider, die sich in knapp der Hälfte der Fälle als unsicher bei der Diagnostik, bei der Therapie und insbesondere bei der Überweisung von $\mathrm{Pa}$ tienten beurteilen.

Bemerkenswert ist im Zusammenhang mit den Einstellungen auch, daß die Zuständigkeit für Angststörungen offensichtlich eher bei Psychotherapeuten und Psychologen gesehen

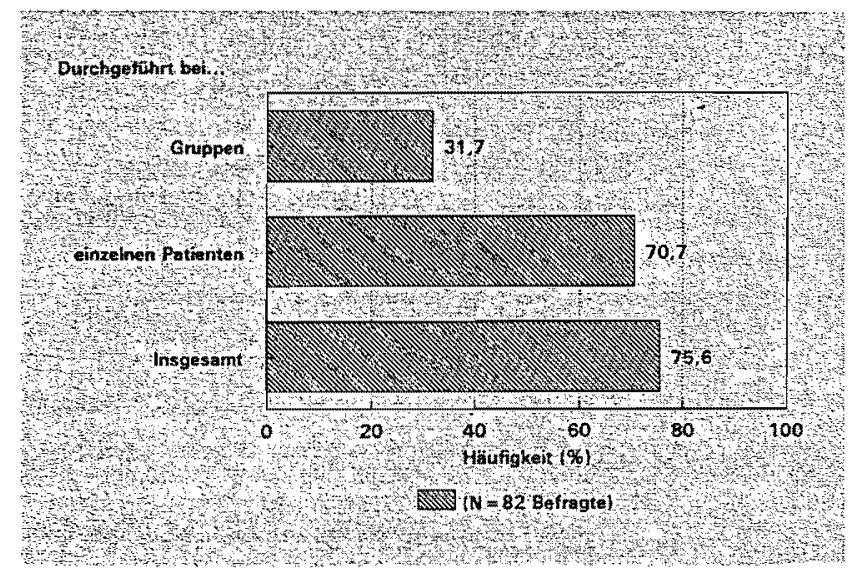

Abb. 4. Wie hăufig haben die Teilnehmer das Patientenseminar durchgeführt? - 3 Monate später - 


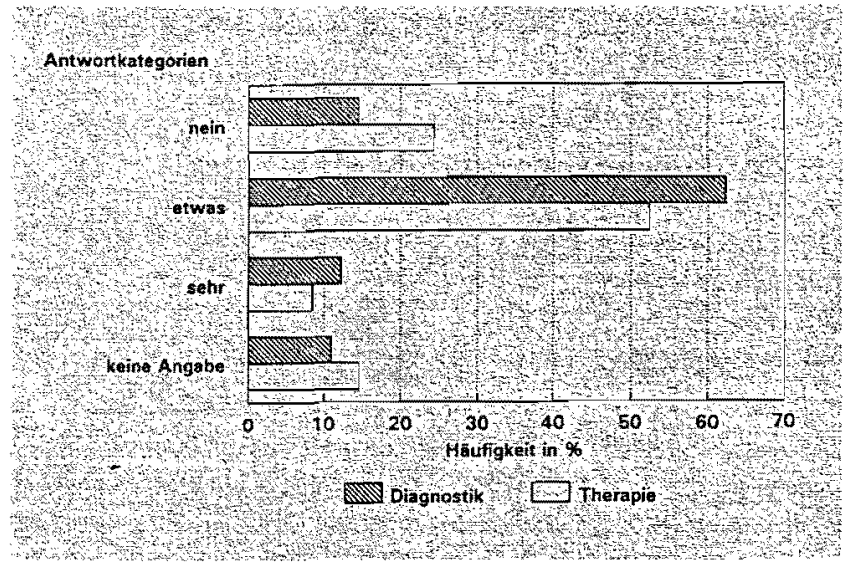

Abb. 5. Effektivităt des Patientenseminars: Sind Sie sicherer in Diagnostik und Therapie?

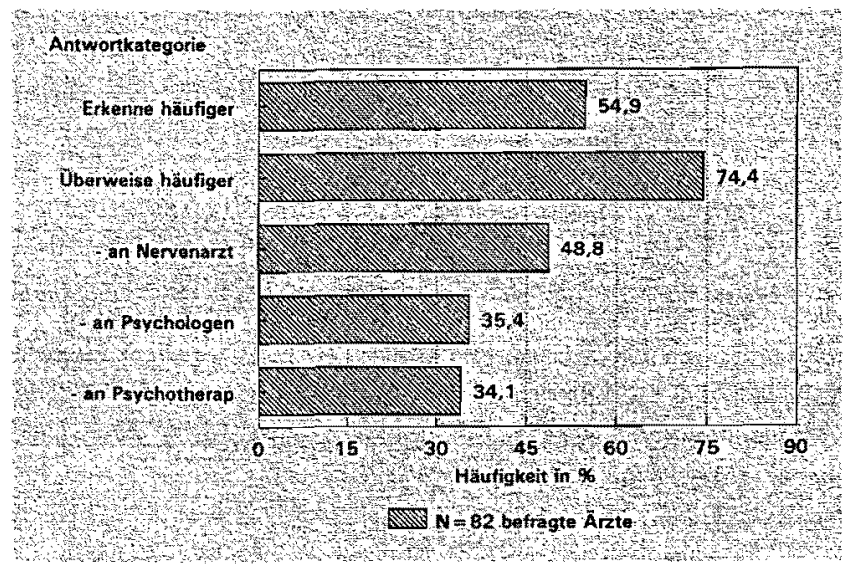

Abb. 6. Erkennen und überweisen Sie hâufiger als früher Angstpatienten?

wird und zumindest vor der Durchführung des Patientenseminars Psychiater in der Überweisungstendenz deutlich niedriger liegen.

Die von uns gezeigten Effekte des Patientenseminars sind auf der diagnostischen Ebene auf den ersten Blick marginal. Der Zuwachs an richtigen Diagnosen betrug bei der einfachen Phobie $12 \%$, bei der Panikstorung lediglich $6 \%$, und nur bei der posttraumatischen Belastungsstörung ist eine Verbesserung um knapp $15 \%$ zu konstatieren. Vorbehaltich differenzierterer Auswertungen könnte diese Veränderungsrate andeuten, daß zum einen die Grenzen differentialdiagnostischer Erwägungen in der Allgemeinarztpraxis eng gesteckt sind und daß möglicherweise eine wesentliche Verbesserung des Erkennens von Angsterkrankungen auf differentialdiagnostischer Ebene nicht möglich ist. Andererseits könnte auch zu erwägen sein, durch Intensivierung von diagnostischen Fallbeispielen das Seminar dahingehend zu verbessern, daß ein weiteres Spektrum differentialdiagnostischer Erwägungen Eingang in die Uberlegungen von Allgemeinärzten findet.

Vom Ausmaß her unerwartet für uns war die sehr große Akzeptanz des Patientenseminars. Dabei ist zu berükssichtigen, daß das Patientenseminar weder bei der Durchführung mit einzelnen Patienten noch auf der Gruppenebene abrechenbar war. Trotzdem haben ein Drittel aller Teilnehmer es in Gruppen durchgeführt, und bei über $70 \%$ wurden die Unterlagen zur Information einzelner Patienten verwandt. Versorgungsbezogen heißt das, $\mathrm{da} B$ in der dreimonatigen Follow-up-Untersuchung über 350 Patienten mit den Inhalten des Patientenseminars konfrontiert wurden. Es kann nur spekuliert werden, daß hierdurch eine Reihe von positiven Effekten bezüglich einer adäquateren Einstellung gegenüber den betroffenen Angstpatienten initiiert wurde. Überrascht waren wir auch bezüglich der außerordentlich positiven Befunde bezüglich des häufigeren Erkennens von Angststörungen, wobei angesichts der nach wie vor hohen Fehlzuteilungsrate diagnostischer Art hinterfragt werden muß, wie hoch der Prozentsatz vollkommen falsch klassifizierter Patienten dabei ist.

Entgegen der Erwartung vieler Kollegen ist auch keineswegs zu befürchten, daß die überreichten Unterlagen zu einer extremen Intensivierung eigener therapeutischer Bemühungen bei Allgemeinärzten führen. $74,4 \%$ geben an, häufiger als vor Durchführung des Seminars ihre Patienten an andere, insbesondere an Spezialisten, zu überweisen, wobei gerade die vor der Durchführung des Patientenseminars niedrige Überweisungsrate an Nervenärzte eine wesentliche Verbesserung erfuhr. $48,8 \%$ gaben an, nun häufiger an den Nervenarzt $z u$ überweisen. Besonders erfreulich ist auch, daß Allgemeinärte offensichtlich verstärkt an Psychologen und Psychotherapeuten überweisen. Dies korrespondiert auch mit ihrer initialen Einstellung, daß für diese Störungsbilder Psychologen und Psychotherapeuten eine vorrangige Therapieentscheidungsmaßnahme darstellen.

Zusammenfassend konnten in der ersten vorläufigen Auswertung eindrucksvolle Belege für die Akzeptanz und in einigen Bereichen auch überraschend für eine hohe Effektivität der Fortbildungsmaßnahmen gesammelt werden. In weiteren Evaluationsschritten beabsichtigen wir nun, differenzierter Teilgruppen von Ärzten mit unterschiedlicher Praxiserfahrung, unterschiedlicher Ausrichtung und unterschiedlichen Versorgungsleistungen im Bereich von Angststörungen zu analysieren. Gleichzeitig erhoffen wir uns auch genauere Aufschlüsse von der Auswertung anderer Kenntnis- und Wissenskategorien in ihren Wechselwirkungen mit Einstellungen gegenüber Angstpatienten.

\section{Literatur}

Margraf J, Schneider S: Panik: Angstanfälle und ihre Behandlung Berlin, Heidelberg, Springer, 1989.

McGlynn TJ, Metcalf HL: Diagnostik und Therapie von Angststörungen. Ein Handbuch für den Arzt. Übersetzung von: Diagnosis and Treatment of Anxiety Disorders: A Physician's Handbook. Urheberrecht: American Psychiatric Press Inc. Heppenheim Upjohn $\mathrm{GmbH}, 1993$.

Sartorius $\mathrm{N}_{2}$ Goldberg D, de Girolamo G, Costa e Silva JA, Lecrubier Y, Wittchen H-U (eds): Psychological Disorders in General Medical Settings. Toronto, Hogrefe \& Huber, 1990.

Sartorius N, Üstün TB, Costa e Silva JA, Goldberg D, Korff M von, Lecrubier Y, Ormel H, Wittchen H-U: Mental Illness in General Health Care Across the World: An International Study. New York, NY, Wiley, im Druck. 
Wittchen $\mathrm{H}-\mathrm{U}$, Bullinger-Naber M, Hand I, Kasper S, Katschnig H, Linden M, Margraf J, Möller H-J, Naber D, Pöldinger W: Patientenseminar Angst. Wie informiere ich meine Patienten über Angst? Basel, Freiburg, Karger, 1993.

Wittchen H-U, Bullinger-Naber M, Hand I, Kasper S, Katschnig H, Linden M, Margraf J, Möller H-J, Naber D, Pöldinger W: Ratgeber Angst. Was Sie schon immer über Angst wissen wollten. Basel, Freiburg, Karger, 1993.

Wittchen H-U, Essau CA, Zerssen D von, Krieg Cl, Hecht H: Lifetime and six-month prevalence of mental disorders in the Munich
Follow-up Study. European Archives of Psychiatry and Clinical Neurosciences 1992;241:247-258.

Sonderdruckbestellungen an:

Prof. Dr. Hans-Ulrich Wittchen

Max-Planck-Institut für Psychiatrie

Klinisches Institut, Klinische Psychologie

Kraepelinstraße. 2

D-80804 München (BRD) 